\title{
1 Small Molecule Modulation of Microbiome Ecosystem: A Systems
}

\section{Pharmacology Perspective}

3 Qiao Liu ${ }^{1}$, Bohyun Lee ${ }^{2}$, Lei Xie Xi,2,3,4,* $^{1}$

$5 \quad{ }^{1}$ Department of Computer Science, Hunter College, The City University of New York

$6 \quad{ }^{2} \mathrm{Ph} . \mathrm{D}$. Program in Computer Science, The City University of New York

$7 \quad{ }^{3}$ Ph.D. Program in Biochemistry and Biology, The City University of New York

$8 \quad{ }^{4}$ Helen and Robert Appel Alzheimer's Disease Research Institute, Feil Family Brain \& Mind

9 Research Institute, Weill Cornell Medicine, Cornell University

10

$11 *$ Correspondence should be addressed

12

13 Short Title: Small Molecule Modulation of Microbiome Ecosystem

14 


\section{Abstract}

21 An increasing body of evidence suggests that microbes are not only strongly associated with many

22 human diseases but also responsible for the efficacy, resistance, and toxicity of drugs. Small-

23 molecule drugs which can precisely fine-tune the microbial ecosystem on the basis of individual

24 patients may revolutionize biomedicine. However, emerging endeavors in small-molecule

25 microbiome drug discovery continue to follow a conventional "one-drug-one-target-one-disease"

26 process. It is often insufficient and less successful in tackling complex systematic diseases. A

27 systematic pharmacology approach that intervenes multiple interacting pathogenic species in the

28 microbiome, could offer an attractive alternative solution. Advances in the Human Microbiome

29 Project have provided numerous genomics data to study microbial interactions in the complex

30 microbiome community. Integrating microbiome data with chemical genomics and other

31 biological information enables us to delineate the landscape for the small molecule modulation of

32 the human microbiome network. In this paper, we construct a disease-centric signed microbe-

33 microbe interaction network using metabolite information of microbes and curated microbe effects

34 on human health from published work. We develop a Signed Random Walk with Restart algorithm

35 for the accurate prediction of pathogenic and commensal species. With a survey on the druggable

36 and evolutionary space of microbe proteins, we find that $8-10 \%$ of them can be targeted by existing

37 drugs or drug-like chemicals and that $25 \%$ of them have homologs to human proteins. We also

38 demonstrate that drugs for diabetes are enriched in the potential inhibitors that target pathogenic

39 microbe without affecting the commensal microbe, thus can be repurposed to modulate the

40 microbiome ecosystem. We further show that periplasmic and cellular outer membrane proteins

41 are overrepresented in the potential drug targets set in pathogenic microbe, but not in the 
42 commensal microbe. The systematic studies of polypharmacological landscape of the microbiome

43 network may open a new avenue for the small-molecule drug discovery of microbiome.

44

45

46

47

48

49

50

51

52

53

54

55

56

57

58

59

60

61

62

63

64

65

66

67

68

69

70

71 


\section{Author Summary}

73 As one of the most abundant components in human bodies, the microbiome has an extensive

74 impact on human health. Pathogenic-microbes have become emerging potential therapeutic targets.

75 Small-molecule drugs that only intervene in the growth of a specific pathogenic microbe without

76 considering the interacting dynamics of the microbiome community may disrupt the ecosystem

77 homeostasis, thus can cause drug side effect or prompt drug resistance. To discover novel drugs

78 for safe and effective microbe-targeting therapeutics, a systematic approach is needed to fine-tune

79 the microbiome ecosystem. To this end, we built a disease-centric signed microbe-microbe

80 interaction network which accurately predicts the pathogenic or commensal effect of microbe on

81 human health. Based on annotated and predicted pathogens and commensal species, we performed

82 a systematic survey on therapeutic space and target landscape of existing drugs for modulating the

83 microbiome ecosystem. Enrichment analysis on potential microbe-targeting drugs shows that

84 drugs for diabetes could be repurposed to maintain the healthy state of microbiome. Furthermore,

85 periplasmic and cellular outer membrane proteins are overrepresented in the potential drug targets

86 of pathogenic-microbes, but not in proteins that perturb commensal-microbes. Our study may open

87 a new avenue for the small molecule drug discovery of microbiome. 


\section{Introduction}

93 As the most abundant organism, symbiotic microbiome biomasses in human body sites are as

94 rich as the human somatic cells [1]. Traditional culture-based or non-culture-based methods only

95 detect limited groups of microbes, restricting our scopes on a comprehensive understanding of

96 the entire microbial community. Advances in high throughput sequencing technology

97 substantially enhance our powers to characterize the microbial community. Up to date, thousands

98 of microbe genomes have been sequenced [2]. These large scale sequencing data collected have

99 driven forward a myriad of intriguing researches, including finding microbiome biomarkers [3,

$1004]$, investigating their association with diseases [5, 6], and uncovering the dynamicity of

101 microbial community $[7,8]$.

102

103 Many human genome biomarkers have been identified and utilized in disease diagnosis and

104 precise treatment [9]. For example, small molecular drug discovery on individual cancer

105 biomarkers has led to successful therapies [9]. Microbiome, as the "forgotten organ" of humans,

106 contains more numerous and more diverse genomic information than the human genome. It has

107 been shown that small molecule drugs, like antibiotics, relieve bacterial infection symptoms by

108 controlling the overgrowth of pathogens [10]. However, many microbe species have developed

109 antibiotics resistance mechanism, especially to several widely used drugs $[11,12]$. This raises the

110 requirement for new drugs targeting on microbes. The most direct way of drug discovery via

111 microbe targeting is to identify drug candidates that modulate or disrupt targeted microbe

112 proteins. The concern that comes with drug intervention treatment is their adverse effect $[13,14]$.

113 Drug intervention causes microbiota compositional change. The current view believes that

114 microbiota homeostasis is a crucial healthy feature of our "forgotten organ"[15]. Elimination or 
115 diminution of healthy commensal microbes draws dysbiosis in our body site ecologically, and

116 then causes symptoms like diarrhea and nausea [13]. Thus drugs minimizing side effects on other

117 symbiotic microbes are desired.

119 Current studies further reveal that the microbial community is associated with a large variety of

120 human phenotypes and diseases $[5,6]$. As the microbes co-evolved with humans, many of them

121 form mutualistic interaction with the host. Microbiota on colon mucus degrades macromolecules

122 to metabolites, which can be digested by human colonocytes [16]. The microbes can also assist

123 human immune system development [17]. Commensal species in human skins and colon sites

124 expressed anti-microbial peptides (AMP) to protect against pathogenic species, like

125 Staphylococcus aureus [18]. Akkermansia emuciniphila improves cancer immunotherapy

126 efficiency [19]. Intervention on microbial community can potentially cause outgrowth of

127 pathogenic species or suppression of commensal species, disrupting the homeostasis between

128 microbiome and host cells and leading to diseases, including obesity [20], allergy [21], type 1

129 diabetes (T1D) [22] and type 2 diabetes(T2D) [23], inflammatory bowel disease (IBD) [24],

130 rheumatoid arthritis (RA) [25], autism [26] and cancer [27]. For instance, T1D studies have

131 shown that the abundance of Bacteroides in patient group is higher than that in the control group

$132[22]$.

134 Symbiotic microbiota in the human body is an ecosystem. Hundreds of microbes coexist in

135 different human body sites. The transition from healthy state to disease state often results from

136 the disruption of microbiome system. Thus fine-tuning the microbiome ecosystem to maintain or

137 to restore its healthy state could be effective strategy for the prevention and treatment of 
138 diseases. Existing efforts in microbiome therapeutics are dominated by probiotics or prebiotics.

139 Despite their success in treating sudden dysbiosis like $C$. difficile infection, phenotypic responses

140 of probiotics and prebiotics are less predictable because they change the entire microbial taxa in

141 an unspecific way. For chronic degenerative diseases, it is needed to precisely fine-tune

142 microbial metabolomics on the basis of individual patients. To this end, small-molecule drugs

143 offer new opportunities and has emerged as a new frontier for microbiome drug discovery and

144 precision medicine [28]. However, emerging endeavors in small-molecule microbiome drug

145 discovery continue to follow a conventional "one-drug-one-target-one-disease" process. It is

146 often insufficient and less successful in tackling complex systematic diseases. Systems

147 pharmacology, which aims to modulate multiple targets of interacting microbiome-microbiome

148 network, could be a potentially powerful approach to microbiome drug discovery. In order to

149 realize systems pharmacology of microbiome, many unanswered questions remain: if there are

150 distinct communities in the interspecies interaction network between the pathogenic

151 microbiomes and commensal microbiomes? if we can target multiple pathogenic microbiomes, at

152 the same time not inhibit commensal microbiomes? what the chemical space is in which the

153 chemical compounds will inhibit pathogenic microbiome interactions but not disturb commensal

154 microbiome? We attempt to address these questions in this perspective.

156 A microbe-microbe interaction network is needed for the microbiome systems pharmacology.

157 There are many approaches to construct a microbe-microbe network based on existing evidence.

158 The most common method is to infer this network based on microbiome occurrence abundance

159 in the host $[29,30]$. Due to the appearance of advanced sequencing technology and

160 metagenomics, getting microbiome abundance directly from human body sites becomes 
161 available. Studies inferring the microbe-microbe interaction network is based on the abundance

162 correlation between microbes, such as Spearman or Pearson correlation methods [31-33].

163 However, the microbe occurrence abundances are mostly compositional. It is still a big challenge

164 to get absolute abundance. Plenty of research is dedicated to overcoming this problem [34, 35].

165 Another network inference approach is to detect microbe interactions using longitudinal data.

166 The network generated using this method is directed and can demonstrate more various

167 interaction types [36, 37]. However, it still suffers many issues, such as hard to get biological

168 meaning and the requirement of optimization of sampling strategies $[38,39]$. 
170 Here we constructed a disease-centric gut microbial community network by inferring microbe-

171 microbe relationship from their metabolites input and output profiles [40], annotating microbe

172 node in the network on their effects on human health by manually literature review, and predicting

173 potential pathogenic and commensal microbiomes using a new signed Random Walk with Restart

174 algorithm. We performed a survey on current knowledge of drugs-targets interactions and found a

175 significant number of genes that could potentially be drug targets in each microbe. Our analyses

176 suggested that large amount of genes have homologs to existing drug targets. Potential drugs,

177 which target on pathogenic microbes with fewer side effects on the commensal microbes, can be

178 identified. We also identified a list of potential protein targets in the pathogenic microbe and not

179 in the commensal microbe. Our analyses took into account of the inherent factors of how drugs

180 affect microbe growth and how microbiome interplays with each other at the molecular level. This

181 application is not limited to the exemplar analysis performed here. It also shows the potential to

182 filter out small molecular drugs targeting distinct pathogenic microbes based on specific patient

183 microbiome signature. The systematic studies of polypharmacological landscape of microbiome

184 network may open a new avenue for the small molecule drug discovery of microbiome.

\section{Results}

\section{A novel disease-centric microbe-microbe interaction network}

188 We here proposed a new microbe-microbe interaction network, which is inferred from each

189 microbe's metabolite consumption and production profile. Microbes affect each other through

190 different mechanisms. 1) They have negative effects on each other through competing for the

191 same metabolite resources (Figure 1A). 2) One microbe can have positive effects on others

192 through cross-feeding (Figure 1A). 3) They can affect other microbes positively or negatively by 
193 alternating their living environment, like the change of $\mathrm{pH} .4$ ) They could also form predator-

194 prey relationships. We can well characterize the first two relationships between microbes

195 through inferring an interaction network using microbes' metabolite consumption and production

196 profiles (Figure 1B). To be specific, the extent of negative relationship is calculated as the

197 Jaccard similarity of two microbes metabolite consumption profiles (Figure 2A). Intuitively, the

198 more metabolites two microbes consume in common, the higher the negative effect they have on

199 each other. On the other side, the positive effect is due to the cross-feeding relationship. The

200 extent of positive effect is calculated as the Jaccard similarity of one microbe's production profile

201 and the other's consumption profile (Figure 2B). It is worth mentioning that a positive

202 relationship between the two microbes is not symmetrical. Finally, the extents of positive effect

203 and negative effect are summarized to generate the final edge weights.

205 Figure 1. Illustration of relationships between microbiomes. A) Microbe A and B compete

206 for metabolite a and has a negative effect on each other. This negative relationship is shown with

207 a red arrow. Microbe $\mathbf{A}$ and microbe $\mathbf{C}$ have a cross-feeding relationship. Microbe $\mathbf{A}$ can degrade

208 macromolecule a into metabolite $\mathbf{c}$, which can be taken by microbe $\mathbf{C}$. Cross-feedings also exist

209 between microbe $\mathbf{B}$ and $\mathbf{C}$, and between microbe $\mathbf{B}$ and $\mathbf{D}$ through metabolite $\mathbf{c}$ and $\mathbf{d}$,

210 respectively. B) An example for the calculation of the relationship between two microbes.

211 Negative effects are calculated as the Jaccard similarity between microbes consumption profiles.

212 The positive effect is calculated as the Jaccard similarity of one microbe's consumption profile

213 and another's production profile. The final effect is the aggregation of the negative effect and the

214 positive effect. 
217 Figure 2. The jaccard similarity among microbes' metabolite consumption and production

218 profiles. A) microbe-microbe negative effect score matrix computed as the Jaccard similarity of

219 their metabolite consumption profiles. B) microbe-microbe positive effect score matrix

220 computed as the Jaccard similarity of one microbiome's metabolite consumption profile and

221 another's metabolite production profile.

222

223 The microbe's metabolite consumption and production profiles were curated elsewhere [40]. We

224 include 512 microbe nodes into our network (Figure 3A). This network has the following merits:

225 1) The graph is directed. Therefore it can thoroughly represent various types of microbe-microbe 226 relationships. The relationships are not limited to competition (++) and mutualism(--), where

227 microbes can positively affect each other and negatively affect each other in both directions

228 (Figure 3C). "+" or "-“" indicates that the microbe has a positive or negative effect on the other in 229 one direction, respectively. It can also represent more diverse relationships, including the 230 commensalism $(+0)$, parasitism $(+-)$ and amenalism $(-0) .0$ here indicates that no relationship is

231 found in a specific direction. 2) It is biologically meaningful and straightforward to interpret. 3)

232 This microbe-microbe interaction network can avoid the problem in the construction of

233 microbiome network based on the abundance correlation of microbiomes, such that the

234 correlation is sensitive to the data compositionality and is affected by low-abundance [41, 42]. 4)

235 It can be integrated with additional networks that are derived from other information (e.g.

236 environmental factors) into a more sophisticated heterogeneous network analysis framework. 
Figure 3. Illustration of Microbe-microbe network. A) Negative relationships (red edges)

240 between microbes. Only the edges with weight lower than -0.7 are shown for simplicity. B)

241 Positive relationships (green edges) between microbes. Only the edges with a weight higher than

2420.5 are shown for simplicity. C) 5 relationships between microbes, competition (--), mutualism

$243(++)$, commensalism(+0), parasitism (+-) and amenalism(-0) are shown.,-+ and 0 denotes a

244 negative effect, a positive effect, and no effect, respectively. pathogenic and commensal

245 microbes curated through literature review are labeled in red and green, respectively. Other

246 microbes are labeled in gray.

248 As the "forgotten organ" of the human body, the microbiome has various effects on human

249 health. We manually curated the microbes' influence on human health through literature review.

250 Out of 513 microbes in our network, we curated 72 microbes having pathogenic effects on

251 human health and 32 microbes having commensal effects on human health (Supplementary

252 Table S1 and Supplementary Table S2). The rest microbe influences on human health are not

253 determined. The reasons are either lack of evidence or hard to determine the influences. Some

254 species has different strains which have different influence on human health [43]. The microbe

255 effects on human health are also integrated into an interaction network. The commensal microbe

256 nodes have node weighted as "+1", while the pathogenic microbe nodes have node weighted as

257 "-1". Other nodes are left unlabeled. The following analysis was performed using this network. 
261 Because most microbe effects on human health are unknown, we developed a graph mining

262 strategy to infer their effects based on annotated network. Using a Signed Random Walk with

263 Restart (SRWR) model, each unannotated microbial species was treated as a node with an

264 unknown weight, and tested for how it was influenced by the neighboring nodes in the network

265 through corporation (positive signed edge) and competition (negative signed edge). The

266 advantage of incorporating SRWR model into our analysis was on the fact that the network

267 recognizes both cooperative relationships as well as the competitive relationships, which

268 resembles the true nature of the microbial ecology. The premise of our analysis is that "friend" of

269 "friend" or "enemy" of "enemy" will be "friend", and "friend" of "enemy" or "enemy" of

270 "friend" will be "enemy".

272 Our SRWR simulation yielded the prediction of 143 positive nodes (potentially commensal) and

273265 negative nodes (potentially pathogenic) for 418 total species without annotations associated 274 with human health (Supplementary material Table S3). To assess the accuracy of our predictions, 275 we use the curated data set of the 32 commensal and 72 potential pathogenic bacterial species

276 that affects human health as a benchmark. We obtained confusion matrix with an average F1

277 score of 0.905 . Specifically, the prediction of positive nodes yielded the precision of 0.780 and

278 the recall of 1.000, while the prediction of negative nodes yielded the precision of 1.000 and the 279 recall of 0.875

281 Survey on microbe proteins druggability and structural predictability 
283 We define a microbe protein to be druggable if drugs or chemicals can target itself or its

284 homolog. To have a comprehensive view of the microbiome, we included all protein sequences

285 from 2232 microbe species collected by the Human Microbiome Project (HMP) in our study [2,

286 44]. Drugbank and ChEMBL databases are two of the most popular and updated drug-target

287 interaction databases $[45,46]$. Up to date, DrugBank and ChEMBL possessed more than 5000

288 and 15500 protein target sequences and drug information interacting with these targets. For each

289 microbe species, we compared its protein sequences with the target sequences in each drug-target

290 interaction database using PSI-Blast[47-49]. We found that a significant amount of proteins in

291 each microbe species have homologs targeted by drugs or drug-like chemicals. The e-value

292 resulting from a specific sequence search indicates the number of hits we can get by chance

293 when we search a protein sequence against a database. From the plot of the percentage of protein

294 with homologs in each microbe versus - log (e-value), we determined that the elbow point of

295 curve is at which e-value is around 10e-60 (Figure 4A). With this e-value, we determined that

$29610 \%$ and $8 \%$ of microbe protein sequences were found to have close homologs in DrugBank and

297 ChEMBL targets database. Besides, the structure information of protein is critical for the

298 structure-based drug design and polypharmacology [50]. We searches for the homologs, which

299 show high sequence similarities with microbe proteins, in Protein Data Bank archive (PDB) [51].

300 With e-value at $10 \mathrm{e}-60$, we show that $25 \%$ of microbe proteins have close homologs in PDB

301 (Figure 4B).

303 Figure 4. Percentage of protein targets in microbiomes that have sequence homologs in

304 different databases, A) ChEMBL and Drugbank, and B) PDB. E-value is the criterion used in

305 the sequence similarity search by BLAST. The lower e-value is, the closer homolog is. 


\section{Drugs for Diabetes' potential on drug repurposing}

308 A successful treatment for human diseases caused by microbe infection is antibiotic intervention,

309 which is used to control the overgrowth of one or a group of pathogenic microbes. Due to the

310 overuse of them, many microbes have shown antibiotic resistance [11]. Another problem with

311 some existing drugs is side effects on other symbiotic microbe species, which causes microbiome

312 dysbiosis. Thus, disrupting pathogen interaction network by targeting multiple pathogenic

313 microbiomes but not disturbing commensal microbiomes will be a potential powerful strategy for

314 microbiome drug discovery. Because drug repurposing exhibits more advantages than

315 developing a novel drug [52], we perform a computational screen on FDA approved or

316 investigational drugs for innovative potential drugs for targeting microbes. To avoid undesirable

317 side effects, the drugs should not affect commensal microbes proteins. With this intuition, we

318 search for drugs that can potentially affect simultaneously multiple pathogenic microbes and

319 avoid undesirable effect on commensal microbes.

321 We performed the screening on two databases: Drugbank and STITCH. Most chemicals in the

322 Drugbank database are drugs that are FDA approved or under investigation, and most of the

323 drug-target interactions have experimental evidence. We collected the drugs that could target

324 proteins that are homologs of pathogenic microbes' proteins and then excluded those targeting on

325 homologs of commensal microbes' proteins. Ultimately, we found 589 drugs that satisfy this

326 constraint (Supplementary Table S4). On the other side, parts of compounds in STITCH are

327 predicted drugs that lack experimental support. STITCH database also possesses predicted drug328 targets interaction for each microbe species. Thus the screen includes both drugs and some non- 
329 drug compounds. Drug-target interactions in the STITCH database have various types, like

330 inhibition, activation, and catalysis. We conducted more specific screening by considering each

331 interaction type, as described in Methods. On average, one third of compounds in the STITCH

332 database are found to have pharmaceutical usage. Finally, we found 170 drugs that appear in

333 both STITCH screen and DrugBank screen results (Supplementary Table S5).

335 We then performed drugs overrepresentation analysis of these 170 drugs. The background drug

336 list used in this analysis includes all drugs targeting microbe proteins homologs. Two analyses

337 are conducted with two different drug classification systems, including the anatomical

338 therapeutic chemical classification system (ATC) and the Drugbank classification system.

339 Surprisingly, both analyses demonstrate that the drugs used in Diabetes are the statistically

340 significantly overrepresented drugs categories (Table 1 and Table 2). Besides, it is also worth

341 noting that the nitric oxide synthases antagonists \& inhibitors are also enriched.

343 Table 1. Hypergeometric test on drugs based on drug category information in Drugbank. Only

344 the categories with Bonferroni corrected p-value, B\&H FDR adjusted p-value, or B\&Y FDR

345 adjusted p-value lower than 0.05 are shown.

\begin{tabular}{ccccc}
\hline & & Bonferroni & B\&H FDR & B\&Y FDR \\
Categories & p-value & corrected p- & adjusted p- & adjusted p- \\
& & value & value & value \\
\hline Nitric Oxide Synthase & 0.00000 & 0.00012 & 0.00012 & 0.00082 \\
antagonists \& inhibitors & & & &
\end{tabular}




\begin{tabular}{|c|c|c|c|c|}
\hline Urea & 0.00000 & 0.00029 & 0.00014 & 0.00094 \\
\hline Sulfonylureas & 0.00004 & 0.01525 & 0.00508 & 0.03327 \\
\hline Oral Hypoglycemics & 0.00007 & 0.02843 & 0.00711 & 0.04652 \\
\hline Drugs Used in Diabetes & 0.00018 & 0.06979 & 0.01204 & 0.07882 \\
\hline Sulfonylurea Compounds & 0.00019 & 0.07226 & 0.01204 & 0.07882 \\
\hline Stereoisomerism & 0.00023 & 0.08839 & 0.01263 & 0.08264 \\
\hline $\begin{array}{l}\text { Aldehyde Reductase, } \\
\text { antagonists \& inhibitors }\end{array}$ & 0.00041 & 0.16059 & 0.02007 & 0.13138 \\
\hline Enzyme Inhibitors & 0.00047 & 0.18206 & 0.02023 & 0.13239 \\
\hline $\begin{array}{c}\text { Blood Glucose Lowering } \\
\text { Agents }\end{array}$ & 0.00061 & 0.23983 & 0.02398 & 0.15696 \\
\hline Pyrazoles & 0.00135 & 0.52458 & 0.04769 & 0.31211 \\
\hline
\end{tabular}

350 Table 2. Hypergeometric test on ATC code information. Only the categories with Bonferroni

351 corrected p-value, B\&H FDR adjusted p-value, or B\&Y FDR adjusted p-value lower than 0.05

352 are shown.

\section{B\&H FDR B\&Y FDR}

Levels $\quad \mathrm{p}$-value Bonferroni adjusted $\mathrm{p}$ - adjusted $\mathrm{p}$ - description value value

$\begin{array}{lllll}\text { A10BB } & 0.00002 & 0.00088 & 0.00088 & 0.00407\end{array}$




\begin{tabular}{|c|c|c|c|c|c|}
\hline A10B & 0.00005 & 0.00298 & 0.00149 & 0.00689 & drugs, excl. insulins \\
\hline A 10 & 0.00013 & 0.00768 & 0.00256 & 0.01185 & $\begin{array}{l}\text { drugs used in diabetes } \\
\text { other blood glucose }\end{array}$ \\
\hline A10BX & 0.00087 & 0.04934 & 0.01234 & 0.05710 & $\begin{array}{l}\text { lowering drugs, excl. } \\
\text { insulins }\end{array}$ \\
\hline A & 0.00158 & 0.09024 & 0.01805 & 0.08355 & $\begin{array}{l}\text { alimentary tract and } \\
\text { metabolism }\end{array}$ \\
\hline
\end{tabular}

\section{Characterization of potential targets in pathogenic microbe proteins}

358 We then identify targets that are homologs of pathogenic microbes' proteins but not those of

359 commensal microbes' proteins. The results can assist in discerning the potential directions in

360 drug discovery. The scope of target identification is limited to the protein targets collected in the

361 Drugbank database. We found 462 potential proteins that are targeted by drugs (Supplementary

362 Table S6). Functional enrichment analysis was then performed on these selected targets with

363 DAVID [53, 54]. The background targets include all found homolog targets of microbes' proteins

364 that are collected by sequence search against the Drugbank database. The results show that

365 proteins in periplasmic and cellular outer membrane are overrepresented (Table 3 ). The

366 statistically significant enriched functional annotations are signal proteins and transport proteins. 
368 Table 3. Results from protein targets functional annotation analysis with DAVID. Only the

369 categories with Bonferroni corrected p-value, B\&H FDR adjusted p-value, or B\&H FDR

370 adjusted p-value lower than 0.05 are shown.

371

\section{B\&H FDR}

\section{B\&Y FDR}

Categories Bonferroni adjusted $\mathrm{p}$ - $\quad$ adjusted $\mathrm{p}$ -

$\begin{array}{cccc} & & \text { value } & \text { value } \\ \text { Periplasm } & 0.00001 & 0.00001 & 0.00007 \\ \text { Signal Peptide } & 0.00010 & 0.00010 & 0.00022 \\ \text { Topological Domain:Periplasmic } & 0.00045 & 0.00022 & 0.00100 \\ \text { GO:0009279 Cell Outer Membrane } & 0.00011 & 0.00011 & 0.00104 \\ \text { Cell Outer Membrane } & 0.00028 & 0.00014 & 0.00133 \\ \text { GO:0030288 Outer Membrane- } & & & 0.00888 \\ \text { Bounded Periplasmic Space } & 0.00097 & 0.00048 & \\ \text { Transmembrane Beta Strand } & 0.00281 & 0.00094 & 0.01348 \\ \text { Signal } & 0.00555 & 0.00139 & 0.02669 \\ \text { Transport } & 0.01127 & 0.00226 & 0.05434\end{array}$

372

373

\section{Discussion}

375 Existing small-molecule microbiome drug discovery follows conventional one-drug-one-gene-

376 one-species paradigm [28], and focuses on preventing infection or fighting against one or a few

377 microbes, barely considering the microbiome as an ecosystem. For examples, antibiotic has a 
378 strong influence on the composition of the microbial community and reduces the diversity of

379 microbiome [20]. Early age onset dysbiosis, caused by antibiotic intake, potentially leads to later

380 emergence of allergy, obesity, IBD, or other disorders. We believe systems pharmacology

381 approaches are necessary to identify small molecule drugs for modulating the microbiome

382 ecosystem instead of targeting a single pathogen. With the awareness of the complexity and

383 integrity of microbiota, the reconstruction of microbiota networks is a critical step to study the

384 microbial community and to realize systems pharmacology, and it draws increasing interests

385 [55]. Besides occurrence abundance correlation-based methods, exploring microbes growth

386 sources and chemical products is crucial to elucidate the mechanism of interplay between

387 microbes.

389 The contemporary medical system undergoes an era of transition from traditional population

390 based diagnosis and treatment to a more precise personalized medicine. Microbiota demonstrates

391 high variability via developing different biogeographic signatures of human body sites [56, 57].

392 Small molecular drug discovery based on patient particular microbiome signatures improves and

393 assists in generating more efficient personalized diagnosis and treatment to cure disease. Our

394 work provides the prime landscape of small molecule drug discovery by exploring the

395 connection between microbe's genome and potential drugs.

397 Our disease-centric microbe-microbe network, constructed based on literature review and 398 computational prediction, is still expected to improve and grow over time. Currently, the label of 399 each node is based on species level. This method introduces ambiguity when defining each 400 species effect on health. For instance, E. coli, as a prevalent commensal species in the human 
401 gut, some strains are pathogenic and even carcinogenic [43]. However, we believe that our

402 network reflects the general effects of microbiota on health, and are useful. Firstly, most of

403 abundant microbiomes, which we include in our network, have been well studied regarding their

404 metabolites and effects on human health. Secondly, rare microbes effect on health inferred with

405 the SRWR method covered the information about how they affect human health by interplaying

406 with abundant microbiomes.

407

408 Another missing piece of information in this work is host and environmental factors. To

409 incorporate host and environmental factors into the construction of a heterogeneous microbe-

410 microbe interaction network will further enhance our understanding of the microbial community.

411 Previous studies showed that environmental factors are another crucial factors determine the

412 diversity and composition of the microbial community [58]. Gut microbiota, as the most

413 abundant microbial community, can be affected by personal daily diet and lifestyle [59]. For

414 example, loss of sleep could increase the ratio of Firmicutes to Bacteroidetes [60]. Microbial

415 community is believed to harbor discrete homeostasis states and transit between different states

416 when experiencing environmental changes, at least for skin or vaginal microbiota [61, 62]. Thus,

417 constructing a heterogeneous human-environment-microbiome network will be an important

418 direction for the future work.

420 Methods

\section{Microbiome interaction network}

422 Microbe species metabolite consumption and production information were manually curated 423 elsewhere [40]. 513 microbe species are included in this dataset. Distribution of the number of 
424 metabolites each microbe consumes or produces, and distribution of the number of microbes

425 each metabolite associates with are investigated (Supplementary Figure 1). We hypothesize that

426 the final relationship between the two microbes is composed of a negative relationship

427 (competition) and a positive relationship (corporation). The negative extent, negative ${ }_{a b}$, is

428 calculated as the Jaccard similarity of metabolite consumption profile between microbe a and

429 microbe b. 233 metabolites are investigated and are consumed by at least one microbe species.

430 The positive extent, positive ${ }_{a b}$, is calculated as the Jaccard similarity of microbiome a's

431 metabolites consumption profile to microbiome b's production profile. At least one microbe

432 species produces 95 metabolites. The final microbiome interaction network is a directed graph.

433 The edge weight between two microbes is calculated as positive $\mathrm{ab}_{\mathrm{ab}}$ - negative ab $_{\mathrm{b}}$

\section{Signed Random Walk with Restart (SRWR)}

436 Microbiome interaction network with selected 512 microbial species were simulated using

437 Signed Random Walk with Restart algorithm [63]. To predict the label (pathogenic or

438 commensal) of a unannotated microbiome species, it was initialized as a start node. For each run,

439 initial score of 1.0 was assigned to the start node with an unknown sign, and then this score was

440 distributed out to the neighboring nodes via edges in the network as the walk goes with random

441 probability. Positive edge would increase the positive ranking score of the neighboring node with

442 the balance attenuation probability of $\beta=0.5$, and the negative edge would increase the negative

443 ranking score of the neighboring node with the probability of $\gamma=0.5$. When the walk was

444 complete, positive scores from known commensal species, and negative scores from known

445 pathogenic species were summed up to predict the label for the unknown start node

446 (supplementary table S1, S2 and S3). 


\section{Microbe proteins druggability survey}

449 Protein sequences of 2232 microbes were downloaded from Human Microbiome Projects (HMP)

$450 \quad[2,44]$. Druggable target sequences were downloaded from DrugBank ( $\underline{w w w . d r u g b a n k . c a})$ and

451 ChEMBL websites (www.ebi.ac.uk/chembl ) $[45,46]$. They were saved as fasta format and

452 reformatted to be a Blast database using PSI-Blast tools [47-49]. Each microbe protein sequences

453 were searched against each target sequence database to find their homologs. Biopython package

454 was used to perform sequence search using PSI-Blast [47-49]. All sequence search results with

455 e-value lower than 10e-4 were saved to generate a plot of percentage of protein with homologs in

456 each microbe versus - $\log$ (e-value). Scripts used for analysis are available in

457 https://github.com/qiaoliuhub/drug_target_analysis_on_microbiome.

\section{Potential drugs screening}

460 Drugbank

461 By using the homolog targets from the sequence search, we collected the drugs that potentially

462 target microbe proteins for each microbe. The drug-target interaction database was downloaded

463 from DrugBank (www.drugbank.ca). All drugs that target pathogenic microbes are gathered into

464 a candidate list, then parts of the drugs in the list are excluded if they can potentially target

465 commensal microbes. 589 drugs in the candidate list were left after screening.

467 STITCH

468 STITCH database was downloaded from http://stitch.embl.de/ [64]. STITCH database has

469 grouped drug-target interactions based on microbe species. These drug-target interactions are 
470 also classified into different types, such as inhibition, activation, or catalysis. We focus on two

471 interaction types: inhibition and activation. Our primary purpose is to screen for FDA approved

472 or investigational drugs, so we excluded non-drugs compounds. We utilized the STITCH drug

473 ID information, which is also the same with PubChem compound ID, to retrieve the

474 pharmaceutical function information from the PubChem database by using PUG REST API and

475 E-utilities tools [65]. We performed the following screen: 1) compounds that activate targets in

476 pathogenic microbes but not activate targets in commensal microbes (134 drugs). 2) compounds

477 that activate targets in pathogenic microbes but not inhibit targets in commensal microbes (431

478 drugs). 3) compounds that inhibit targets in pathogenic microbes but not inhibit targets in

479 commensal microbes (185 drugs). 4) compounds that inhibit targets in pathogenic microbes but

480 not activate targets in commensal microbes (1325 drugs) (Supplementary Table S7).

482 The intersection of Drugbank screening and STITCH screening result

483 The InChIKey information of all drugs found in Drugbank screening was retrieved from

484 Drugbank full database XML file. The InChIKey information of all drugs found in STITCH

485 screening was collected from the Pubchem website using PUG REST API and E-utilities tools.

486 The intersection of these two drugs InChIKey list was found for later analysis.

488 Overrepresentation analysis

489 Drug overrepresentation analysis

490 All drugs ATC code and Drugbank classification information were accumulated from Drugbank

491 full database XML file. These two classification systems have hierarchical structures, and all

492 categories in all levels are included. A hypergeometric test was performed on the Drugbank 
screened 589 drugs list. The Bonferroni correction, Benjamini \& Hochberg'sHochberg's FDR

494 adjustment, and Benjamini \& Yekutieli'sYekutieli's FDR adjustment methods were used to

495 adjust the p-values of these multiple comparisons. $\sim 3700$ drugs, which are found to target at least

496 one microbe protein homolog, are used as background drugs list (Supplementary Table S8).

Protein targets functional enrichment analysis

499462 potential protein targets were filtered out using the Drugbank target sequences database and

500 saved as UniProt accession number. Target's functional enrichment analysis was conducted with

501 the database for annotation, visualization, and integrated discovery (DAVID) [53, 54]. The list of

502462 potential targets' UniProt accession numbers was uploaded to DAVID as a test gene set.

503 Background gene set includes 1700 microbe protein homologs (Supplementary Table S9).

504

505 Acknowledgements

506 This work was supported by Grant Number R01GM122845 from the National Institute of

507 General Medical Sciences (NIGMS) and Grand Number R01AD057555 of National Institute of

508 Aging of the National Institute of Health (NIH) as well as CUNY High Performance Computing

509 Center. The funders had no role in study design, data collection and analysis, decision to publish,

510 or preparation of the manuscript.

\section{Reference}

513 1. Sender R, Fuchs S, Milo R. Are We Really Vastly Outnumbered? Revisiting the Ratio of

514 Bacterial to Host Cells in Humans. Cell. 2016;164(3):337-40. Epub 2016/01/30. doi:

515 10.1016/j.cell.2016.01.013. PubMed PMID: 26824647. 
2. Human Microbiome Project C. Structure, function and diversity of the healthy human microbiome. Nature. 2012;486(7402):207-14. Epub 2012/06/16. doi: 10.1038/nature11234. PubMed PMID: 22699609; PubMed Central PMCID: PMCPMC3564958. 3. Rajpoot M, Sharma AK, Sharma A, Gupta GK. Understanding the microbiome: Emerging biomarkers for exploiting the microbiota for personalized medicine against cancer. Semin Cancer Biol. 2018;52(Pt 1):1-8. Epub 2018/02/10. doi: 10.1016/j.semcancer.2018.02.003. PubMed PMID: 29425888. 4. Dietert RR, Silbergeld EK. Biomarkers for the 21st century: listening to the microbiome. Toxicol Sci. 2015;144(2):208-16. Epub 2015/03/22. doi: 10.1093/toxsci/kfv013. PubMed PMID: 25795652.

5. Jackson MA, Verdi S, Maxan ME, Shin CM, Zierer J, Bowyer RCE, et al. Gut microbiota associations with common diseases and prescription medications in a population-based cohort. Nat Commun. 2018;9(1):2655. Epub 2018/07/10. doi: 10.1038/s41467-018-05184-7. PubMed PMID: 29985401; PubMed Central PMCID: PMCPMC6037668.

530 6. Kho ZY, Lal SK. The Human Gut Microbiome - A Potential Controller of Wellness and Disease. Front Microbiol. 2018;9:1835. Epub 2018/08/30. doi: 10.3389/fmicb.2018.01835. PubMed PMID: 30154767; PubMed Central PMCID: PMCPMC6102370.

7. Thaiss CA. Microbiome dynamics in obesity. Science. 2018;362(6417):903-4. Epub 2018/11/24. doi: 10.1126/science.aav6870. PubMed PMID: 30467161. J Biol Med. 2016;89(3):331-7. Epub 2016/10/05. PubMed PMID: 27698617; PubMed Central PMCID: PMCPMC5045142.

9. Hoelder S, Clarke PA, Workman P. Discovery of small molecule cancer drugs: successes, challenges and opportunities. Mol Oncol. 2012;6(2):155-76. Epub 2012/03/24. doi: 10.1016/j.molonc.2012.02.004. PubMed PMID: 22440008; PubMed Central PMCID: PMCPMC3476506. 10. Wilson ID, Nicholson JK. Gut microbiome interactions with drug metabolism, efficacy, and toxicity. Transl Res. 2017;179:204-22. Epub 2016/09/04. doi: 10.1016/j.trs1.2016.08.002. PubMed PMID: 27591027; PubMed Central PMCID: PMCPMC5718288.

\section{Zaman SB, Hussain MA, Nye R, Mehta V, Mamun KT, Hossain N. A Review on} Antibiotic Resistance: Alarm Bells are Ringing. Cureus. 2017;9(6):e1403. Epub 2017/08/31. doi: 10.7759/cureus.1403. PubMed PMID: 28852600; PubMed Central PMCID: PMCPMC5573035. 12. Zinner SH. Antibiotic use: present and future. New Microbiol. 2007;30(3):321-5. Epub 2007/09/07. PubMed PMID: 17802919.

550 13. Jernberg C, Lofmark S, Edlund C, Jansson JK. Long-term impacts of antibiotic exposure 551 on the human intestinal microbiota. Microbiology. 2010;156(Pt 11):3216-23. Epub 2010/08/14. doi: 10.1099/mic.0.040618-0. PubMed PMID: 20705661.

14. Dudek-Wicher RK, Junka A, Bartoszewicz M. The influence of antibiotics and dietary components on gut microbiota. Prz Gastroenterol. 2018;13(2):85-92. Epub 2018/07/14. doi: 10.5114/pg.2018.76005. PubMed PMID: 30002765; PubMed Central PMCID: PMCPMC6040098.

15. Lloyd-Price J, Abu-Ali G, Huttenhower C. The healthy human microbiome. Genome Med. 2016;8(1):51. Epub 2016/04/29. doi: 10.1186/s13073-016-0307-y. PubMed PMID: 27122046; PubMed Central PMCID: PMCPMC4848870.

560 16. Samuel BS, Hansen EE, Manchester JK, Coutinho PM, Henrissat B, Fulton R, et al. 561 Genomic and metabolic adaptations of Methanobrevibacter smithii to the human gut. Proc Natl 
Acad Sci U S A. 2007;104(25):10643-8. Epub 2007/06/15. doi: 10.1073/pnas.0704189104. PubMed PMID: 17563350; PubMed Central PMCID: PMCPMC1890564.

17. Belkaid Y, Hand TW. Role of the microbiota in immunity and inflammation. Cell. 2014;157(1):121-41. Epub 2014/04/01. doi: 10.1016/j.cell.2014.03.011. PubMed PMID: 24679531; PubMed Central PMCID: PMCPMC4056765.

18. Nakatsuji T, Chen TH, Narala S, Chun KA, Two AM, Yun T, et al. Antimicrobials from human skin commensal bacteria protect against Staphylococcus aureus and are deficient in atopic dermatitis. Sci Trans1 Med. 2017;9(378). Epub 2017/02/24. doi: 10.1126/scitranslmed.aah4680. PubMed PMID: 28228596; PubMed Central PMCID: PMCPMC5600545.

19. Routy B, Le Chatelier E, Derosa L, Duong CPM, Alou MT, Daillere R, et al. Gut microbiome influences efficacy of PD-1-based immunotherapy against epithelial tumors. Science. 2018;359(6371):91-7. Epub 2017/11/04. doi: 10.1126/science.aan3706. PubMed PMID: 29097494.

20. Trasande L, Blustein J, Liu M, Corwin E, Cox LM, Blaser MJ. Infant antibiotic exposures and early-life body mass. Int J Obes (Lond). 2013;37(1):16-23. Epub 2012/08/22. doi: 10.1038/ijo.2012.132. PubMed PMID: 22907693; PubMed Central PMCID: PMCPMC3798029. 21. Fujimura KE, Sitarik AR, Havstad S, Lin DL, Levan S, Fadrosh D, et al. Neonatal gut microbiota associates with childhood multisensitized atopy and T cell differentiation. Nat Med. 2016;22(10):1187-91. Epub 2016/09/13. doi: 10.1038/nm.4176. PubMed PMID: 27618652; PubMed Central PMCID: PMCPMC5053876.

22. de Goffau MC, Luopajarvi K, Knip M, Ilonen J, Ruohtula T, Harkonen T, et al. Fecal microbiota composition differs between children with beta-cell autoimmunity and those without. Diabetes. 2013;62(4):1238-44. Epub 2013/01/01. doi: 10.2337/db12-0526. PubMed PMID: 23274889; PubMed Central PMCID: PMCPMC3609581.

23. Qin J, Li Y, Cai Z, Li S, Zhu J, Zhang F, et al. A metagenome-wide association study of gut microbiota in type 2 diabetes. Nature. 2012;490(7418):55-60. Epub 2012/10/02. doi: 10.1038/nature11450. PubMed PMID: 23023125.

24. Huttenhower C, Kostic AD, Xavier RJ. Inflammatory bowel disease as a model for translating the microbiome. Immunity. 2014;40(6):843-54. Epub 2014/06/21. doi: 10.1016/j.immuni.2014.05.013. PubMed PMID: 24950204; PubMed Central PMCID: PMCPMC4135443.

25. Scher JU, Sczesnak A, Longman RS, Segata N, Ubeda C, Bielski C, et al. Expansion of intestinal Prevotella copri correlates with enhanced susceptibility to arthritis. Elife. 2013;2:e01202. Epub 2013/11/07. doi: 10.7554/eLife.01202. PubMed PMID: 24192039; PubMed Central PMCID: PMCPMC3816614.

26. Kang DW, Adams JB, Gregory AC, Borody T, Chittick L, Fasano A, et al. Microbiota Transfer Therapy alters gut ecosystem and improves gastrointestinal and autism symptoms: an open-label study. Microbiome. 2017;5(1):10. Epub 2017/01/27. doi: 10.1186/s40168-016-02257. PubMed PMID: 28122648; PubMed Central PMCID: PMCPMC5264285.

27. Kostic AD, Chun E, Robertson L, Glickman JN, Gallini CA, Michaud M, et al. Fusobacterium nucleatum potentiates intestinal tumorigenesis and modulates the tumor-immune microenvironment. Cell Host Microbe. 2013;14(2):207-15. Epub 2013/08/21. doi: 10.1016/j.chom.2013.07.007. PubMed PMID: 23954159; PubMed Central PMCID: PMCPMC3772512. 
606

28. Cully M. Microbiome therapeutics go small molecule. Nat Rev Drug Discov. 2019;18(8):569-72. Epub 2019/08/02. doi: 10.1038/d41573-019-00122-8. PubMed PMID: 31367062.

29. Ban Y, An L, Jiang H. Investigating microbial co-occurrence patterns based on metagenomic compositional data. Bioinformatics. 2015;31(20):3322-9. Epub 2015/06/17. doi: 10.1093/bioinformatics/btv364. PubMed PMID: 26079350; PubMed Central PMCID: PMCPMC4795632.

30. Berry D, Widder S. Deciphering microbial interactions and detecting keystone species with co-occurrence networks. Front Microbiol. 2014;5:219. Epub 2014/06/07. doi: 10.3389/fmicb.2014.00219. PubMed PMID: 24904535; PubMed Central PMCID: PMCPMC4033041.

31. Arumugam M, Raes J, Pelletier E, Le Paslier D, Yamada T, Mende DR, et al. Enterotypes of the human gut microbiome. Nature. 2011;473(7346):174-80. Epub 2011/04/22. doi: 10.1038/nature09944. PubMed PMID: 21508958; PubMed Central PMCID: PMCPMC3728647.

32. Barberan A, Bates ST, Casamayor EO, Fierer N. Using network analysis to explore cooccurrence patterns in soil microbial communities. ISME J. 2012;6(2):343-51. Epub 2011/09/09. doi: 10.1038/ismej.2011.119. PubMed PMID: 21900968; PubMed Central PMCID: PMCPMC3260507.

33. Copeland JK, Yuan L, Layeghifard M, Wang PW, Guttman DS. Seasonal community succession of the phyllosphere microbiome. Mol Plant Microbe Interact. 2015;28(3):274-85. Epub 2015/02/14. doi: 10.1094/MPMI-10-14-0331-FI. PubMed PMID: 25679538.

34. Li H. Microbiome, Metagenomics, and High-Dimensional Compositional Data Analysis. Annual Review of Statistics and Its Application. 2015;2(1):73-94. doi: 10.1146/annurevstatistics-010814-020351.

35. Tsilimigras MC, Fodor AA. Compositional data analysis of the microbiome: fundamentals, tools, and challenges. Ann Epidemiol. 2016;26(5):330-5. Epub 2016/06/04. doi: 10.1016/j.annepidem.2016.03.002. PubMed PMID: 27255738.

36. Baksi KD, Kuntal BK, Mande SS. 'TIME': A Web Application for Obtaining Insights into Microbial Ecology Using Longitudinal Microbiome Data. Front Microbiol. 2018;9:36. Epub 2018/02/09. doi: 10.3389/fmicb.2018.00036. PubMed PMID: 29416530; PubMed Central PMCID: PMCPMC5787560.

37. Caporaso JG, Lauber CL, Costello EK, Berg-Lyons D, Gonzalez A, Stombaugh J, et al. Moving pictures of the human microbiome. Genome Biol. 2011;12(5):R50. Epub 2011/06/01. doi: 10.1186/gb-2011-12-5-r50. PubMed PMID: 21624126; PubMed Central PMCID: PMCPMC3271711.

38. Gerber GK. The dynamic microbiome. FEBS Lett. 2014;588(22):4131-9. Epub 2014/03/04. doi: 10.1016/j.febslet.2014.02.037. PubMed PMID: 24583074.

39. Datta MS, Sliwerska E, Gore J, Polz MF, Cordero OX. Microbial interactions lead to rapid micro-scale successions on model marine particles. Nat Commun. 2016;7:11965. Epub 2016/06/18. doi: 10.1038/ncomms 11965. PubMed PMID: 27311813; PubMed Central PMCID: PMCPMC4915023.

40. Sung J, Kim S, Cabatbat JJT, Jang S, Jin YS, Jung GY, et al. Global metabolic interaction network of the human gut microbiota for context-specific community-scale analysis. Nat Commun. 2017;8:15393. Epub 2017/06/07. doi: 10.1038/ncomms15393. PubMed PMID: 28585563; PubMed Central PMCID: PMCPMC5467172. 
652 41. Layeghifard M, Hwang DM, Guttman DS. Disentangling Interactions in the Microbiome:

653 A Network Perspective. Trends Microbiol. 2017;25(3):217-28. Epub 2016/12/06. doi:

654 10.1016/j.tim.2016.11.008. PubMed PMID: 27916383.

655 42. Rottjers L, Faust K. From hairballs to hypotheses-biological insights from microbial

656 networks. FEMS Microbiol Rev. 2018;42(6):761-80. Epub 2018/08/08. doi:

657 10.1093/femsre/fuy030. PubMed PMID: 30085090; PubMed Central PMCID:

658 PMCPMC6199531.

659 43. Martinez-Medina M, Garcia-Gil LJ. Escherichia coli in chronic inflammatory bowel

660 diseases: An update on adherent invasive Escherichia coli pathogenicity. World J Gastrointest

661 Pathophysiol. 2014;5(3):213-27. Epub 2014/08/19. doi: 10.4291/wjgp.v5.i3.213. PubMed PMID:

662 25133024; PubMed Central PMCID: PMCPMC4133521.

663 44. Human Microbiome Project C. A framework for human microbiome research. Nature. 2012;486(7402):215-21. Epub 2012/06/16. doi: 10.1038/nature11209. PubMed PMID:

665 22699610; PubMed Central PMCID: PMCPMC3377744.

666 45. Wishart DS, Feunang YD, Guo AC, Lo EJ, Marcu A, Grant JR, et al. DrugBank 5.0: a

667 major update to the DrugBank database for 2018. Nucleic Acids Res. 2018;46(D1):D1074-D82.

668 Epub 2017/11/11. doi: 10.1093/nar/gkx1037. PubMed PMID: 29126136; PubMed Central PMCID: PMCPMC5753335.

670 46. Gaulton A, Hersey A, Nowotka M, Bento AP, Chambers J, Mendez D, et al. The ChEMBL database in 2017. Nucleic Acids Res. 2017;45(D1):D945-D54. Epub 2016/12/03. doi: 10.1093/nar/gkw1074. PubMed PMID: 27899562; PubMed Central PMCID: PMCPMC5210557. architecture and applications. BMC Bioinformatics. 2009;10:421. Epub 2009/12/17. doi: 10.1186/1471-2105-10-421. PubMed PMID: 20003500; PubMed Central PMCID: PMCPMC2803857. 48. Altschul SF, Madden TL, Schaffer AA, Zhang J, Zhang Z, Miller W, et al. Gapped BLAST and PSI-BLAST: a new generation of protein database search programs. Nucleic Acids Res. 1997;25(17):3389-402. Epub 1997/09/01. doi: 10.1093/nar/25.17.3389. PubMed PMID: 9254694; PubMed Central PMCID: PMCPMC146917.

682 49. Altschul SF, Gish W, Miller W, Myers EW, Lipman DJ. Basic local alignment search tool. J Mol Biol. 1990;215(3):403-10. Epub 1990/10/05. doi: 10.1016/S0022-2836(05)80360-2.

PubMed PMID: 2231712.

50. Xie L, Xie L, Kinnings SL, Bourne PE. Novel computational approaches to polypharmacology as a means to define responses to individual drugs. Annu Rev Pharmacol Toxicol. 2012;52:361-79. Epub 2011/10/25. doi: 10.1146/annurev-pharmtox-010611-134630. PubMed PMID: 22017683.

690 Data Bank. Nucleic Acids Res. 2000;28(1):235-42. Epub 1999/12/11. doi: 10.1093/nar/28.1.235. 691 PubMed PMID: 10592235; PubMed Central PMCID: PMCPMC102472.

692 52. Pushpakom S, Iorio F, Eyers PA, Escott KJ, Hopper S, Wells A, et al. Drug repurposing: progress, challenges and recommendations. Nat Rev Drug Discov. 2019;18(1):41-58. Epub

695 53. Huang da W, Sherman BT, Lempicki RA. Systematic and integrative analysis of large 696 gene lists using DAVID bioinformatics resources. Nat Protoc. 2009;4(1):44-57. Epub

697 2009/01/10. doi: 10.1038/nprot.2008.211. PubMed PMID: 19131956. 
54. Huang da W, Sherman BT, Lempicki RA. Bioinformatics enrichment tools: paths toward the comprehensive functional analysis of large gene lists. Nucleic Acids Res. 2009;37(1):1-13. Epub 2008/11/27. doi: 10.1093/nar/gkn923. PubMed PMID: 19033363; PubMed Central PMCID: PMCPMC2615629.

55. Xiao Y, Angulo MT, Friedman J, Waldor MK, Weiss ST, Liu YY. Mapping the ecological networks of microbial communities. Nat Commun. 2017;8(1):2042. Epub 2017/12/13. doi: 10.1038/s41467-017-02090-2. PubMed PMID: 29229902; PubMed Central PMCID: PMCPMC5725606.

56. Hannigan GD, Duhaime MB, Koutra D, Schloss PD. Biogeography and environmental conditions shape bacteriophage-bacteria networks across the human microbiome. PLoS Comput Biol. 2018;14(4):e1006099. Epub 2018/04/19. doi: 10.1371/journal.pcbi.1006099. PubMed PMID: 29668682; PubMed Central PMCID: PMCPMC5927471.

57. Oh J, Byrd AL, Deming C, Conlan S, Program NCS, Kong HH, et al. Biogeography and individuality shape function in the human skin metagenome. Nature. 2014;514(7520):59-64. Epub 2014/10/04. doi: 10.1038/nature13786. PubMed PMID: 25279917; PubMed Central PMCID: PMCPMC4185404.

58. Goodrich JK, Waters JL, Poole AC, Sutter JL, Koren O, Blekhman R, et al. Human genetics shape the gut microbiome. Cell. 2014;159(4):789-99. Epub 2014/11/25. doi: 10.1016/j.cell.2014.09.053. PubMed PMID: 25417156; PubMed Central PMCID: PMCPMC4255478.

59. Gilbert JA, Blaser MJ, Caporaso JG, Jansson JK, Lynch SV, Knight R. Current understanding of the human microbiome. Nat Med. 2018;24(4):392-400. Epub 2018/04/11. doi: 10.1038/nm.4517. PubMed PMID: 29634682.

60. Benedict C, Vogel H, Jonas W, Woting A, Blaut M, Schurmann A, et al. Gut microbiota and glucometabolic alterations in response to recurrent partial sleep deprivation in normalweight young individuals. Mol Metab. 2016;5(12):1175-86. Epub 2016/12/03. doi: 10.1016/j.molmet.2016.10.003. PubMed PMID: 27900260; PubMed Central PMCID: PMCPMC5123208.

61. Ravel J, Gajer P, Abdo Z, Schneider GM, Koenig SS, McCulle SL, et al. Vaginal microbiome of reproductive-age women. Proc Natl Acad Sci U S A. 2011;108 Suppl 1:4680-7. Epub 2010/06/11. doi: 10.1073/pnas.1002611107. PubMed PMID: 20534435; PubMed Central PMCID: PMCPMC3063603.

62. DiGiulio DB, Callahan BJ, McMurdie PJ, Costello EK, Lyell DJ, Robaczewska A, et al. Temporal and spatial variation of the human microbiota during pregnancy. Proc Natl Acad Sci U S A. 2015;112(35):11060-5. Epub 2015/08/19. doi: 10.1073/pnas.1502875112. PubMed PMID: 26283357; PubMed Central PMCID: PMCPMC4568272.

63. Jung J, Jin W, Sael L, Kang U, editors. Personalized Ranking in Signed Networks Using Signed Random Walk with Restart. 2016 IEEE 16th International Conference on Data Mining (ICDM); 2016 12-15 Dec. 2016.

64. Szklarczyk D, Santos A, von Mering C, Jensen LJ, Bork P, Kuhn M. STITCH 5: augmenting protein-chemical interaction networks with tissue and affinity data. Nucleic Acids Res. 2016;44(D1):D380-4. Epub 2015/11/22. doi: 10.1093/nar/gkv1277. PubMed PMID: 26590256; PubMed Central PMCID: PMCPMC4702904.

65. Kim S, Chen J, Cheng T, Gindulyte A, He J, He S, et al. PubChem 2019 update: improved access to chemical data. Nucleic Acids Res. 2019;47(D1):D1102-D9. Epub 


\section{Author contributions}

749 Q.L., B.L. and L.X. designed the computational framework. Q.L. and B.L. carried out the

750 implementation and analyzed the data. Q.L. and B.L. wrote the manuscript with input from all

751 authors. L.X. revised the manuscript, conceived and planned the study.

\section{Competing Interests statement}

754 The authors have declared that no competing interests exist.

\section{Supporting information captions}

757 Supplementary Table S1. Microbes with pathogenic effects on human health by manually

758 literature review.

760 Supplementary Table S2. Microbes with commensal effects on human health by manually

761 literature review.

762

763 Supplementary Table S3. The Microbes with unknown effects in literature reviews and their

764 SRWR inferred microbe effects. 
766 Supplementary Table S4. Drug screen results using Drugbank database.

767

768 Supplementary Table S5. Drugs that are found in both drug screen results using Drugbank

769 database and that using STITCH database.

770

771 Supplementary Table S6. Potential homolog proteins that have homologs with pathogenic

772 microbe proteins but do not have homologs with commensal microbe proteins.

773

774 Supplementary Table S7. Drug screen results in STITCH database.

775

776 Supplementary Table S8. Background drugs list used in drug overrepresentation analysis.

777

778 Supplementary Table S9. Background targets list used in target functional annotation analysis.

779

780 Supplementary Figure 1. A) Distribution of number of metabolites each microbiome consume or

781 produce. B) Distribution of number of microbiomes each metabolite. 


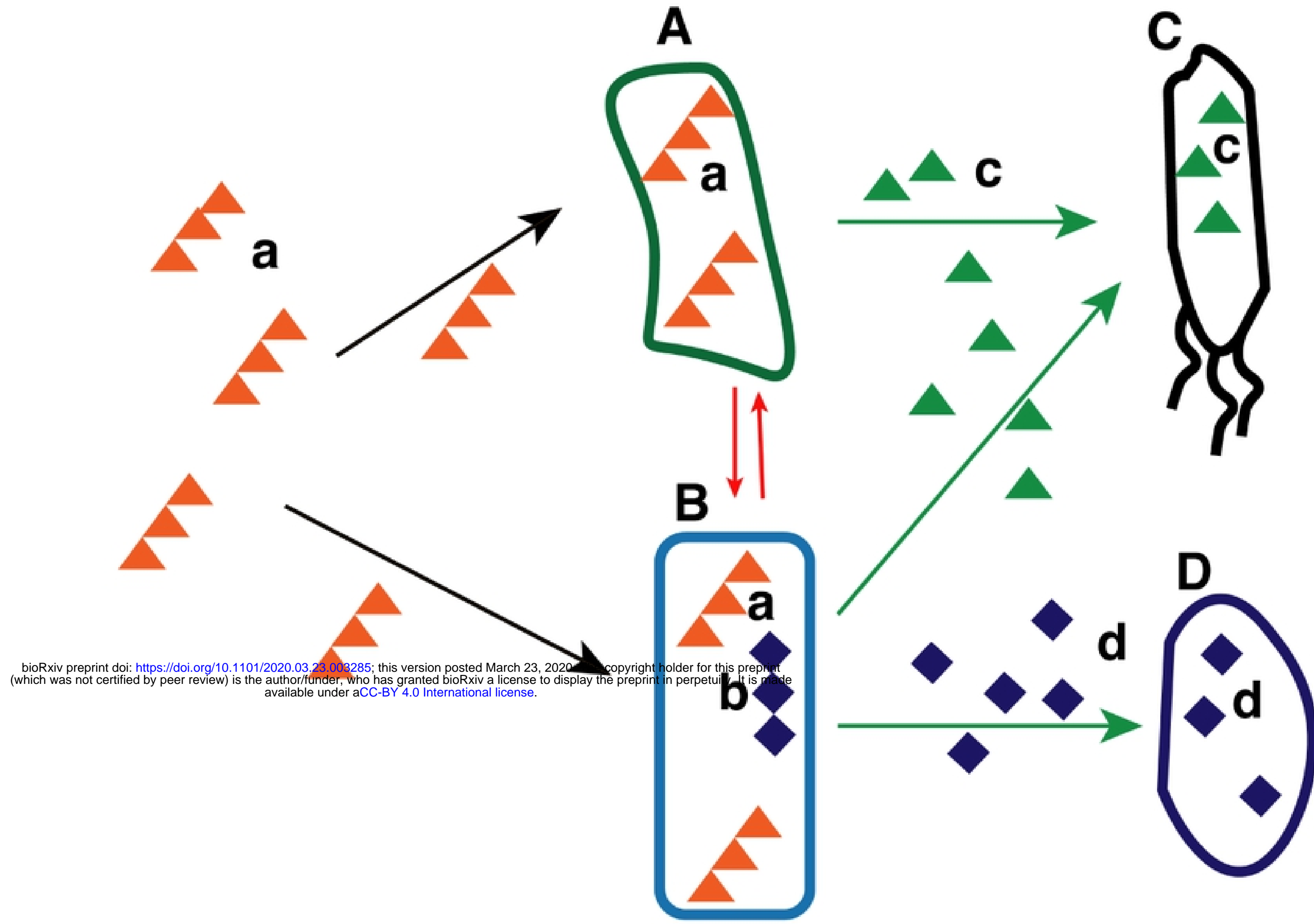

Negative effects

Microbe a consumption profile \begin{tabular}{|l|l|l|l|l|l|l|}
\hline 1 & 0 & 0 & $=-1$ & 1 & 0 \\
\hline
\end{tabular}

Microbe b consumption profile $\left.\begin{array}{l|l|l|l|l|l|l|l|}\hline & 1 & 1 & 0 & =\end{array}\right]$ Jaccard Similarity: 0.5

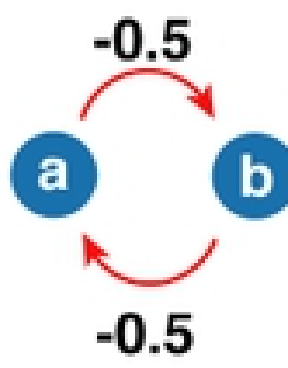

Positive effects

\begin{tabular}{ll|l|l|l|l|l|l|} 
Microbe a production profile & 1 & 0 & 1 \\
\hline
\end{tabular}

Microbe b consumption profile $\left.\begin{array}{l|l|l|l|l|l|l|l|}\hline & 1 & 1 & 0 & = & = & 0 & 0\end{array}\right]$ Jaccard Similarity: 0.33

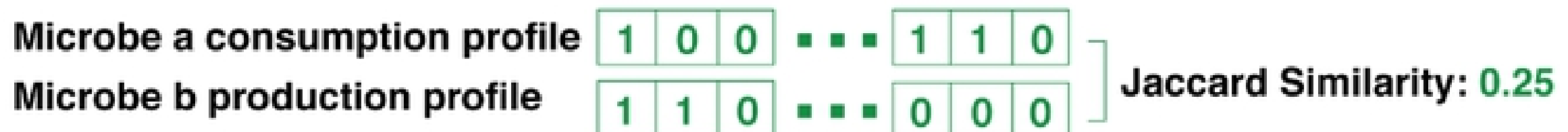
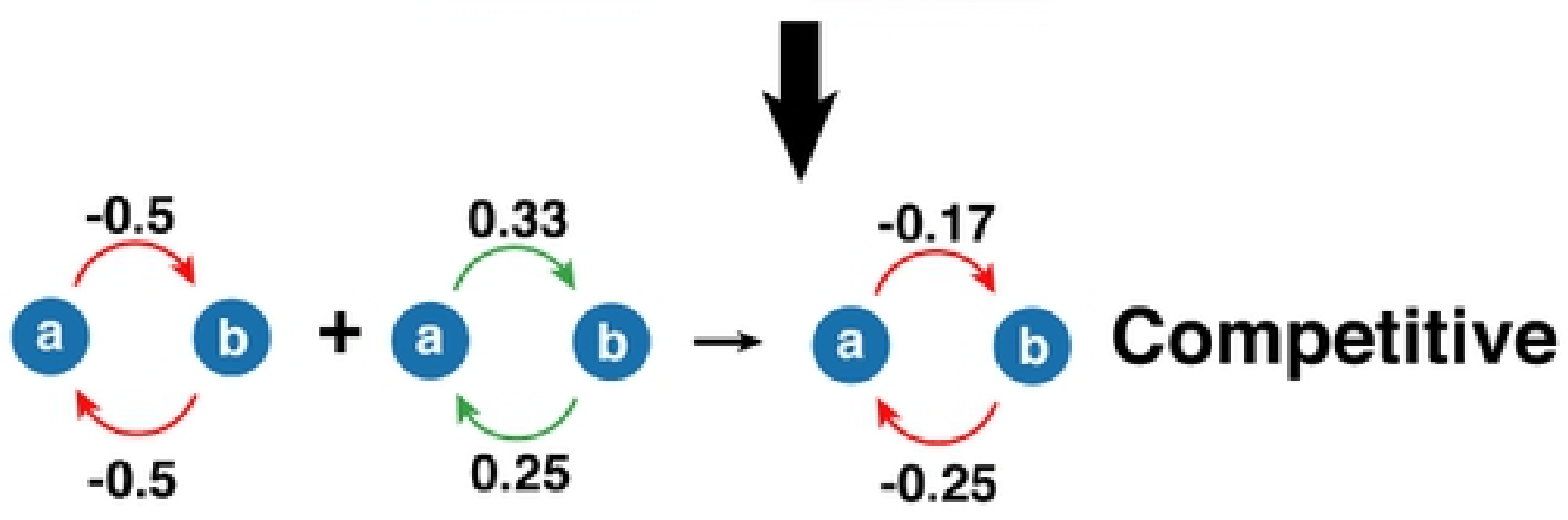

Figure1 
Negative effects

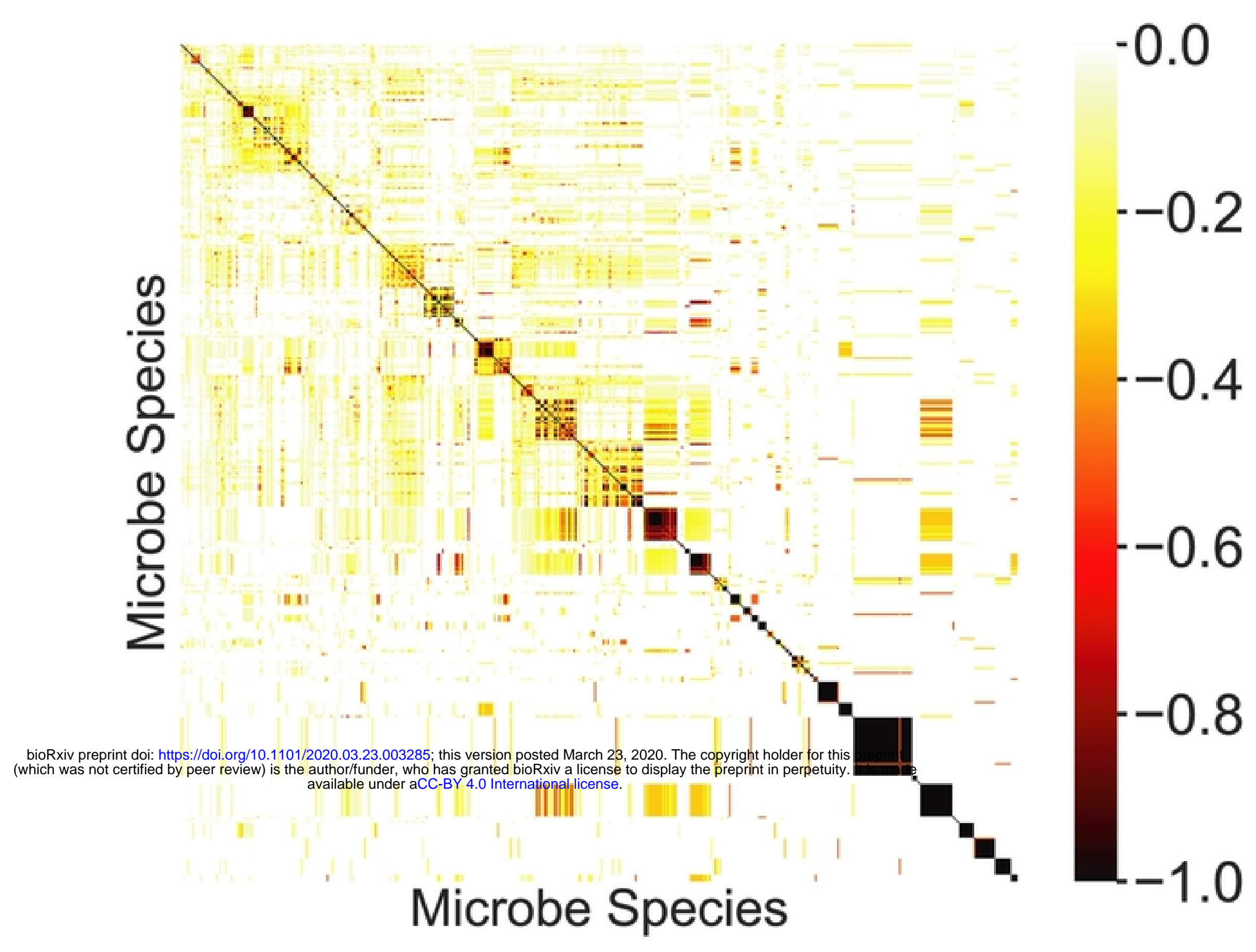

B

Positive effects

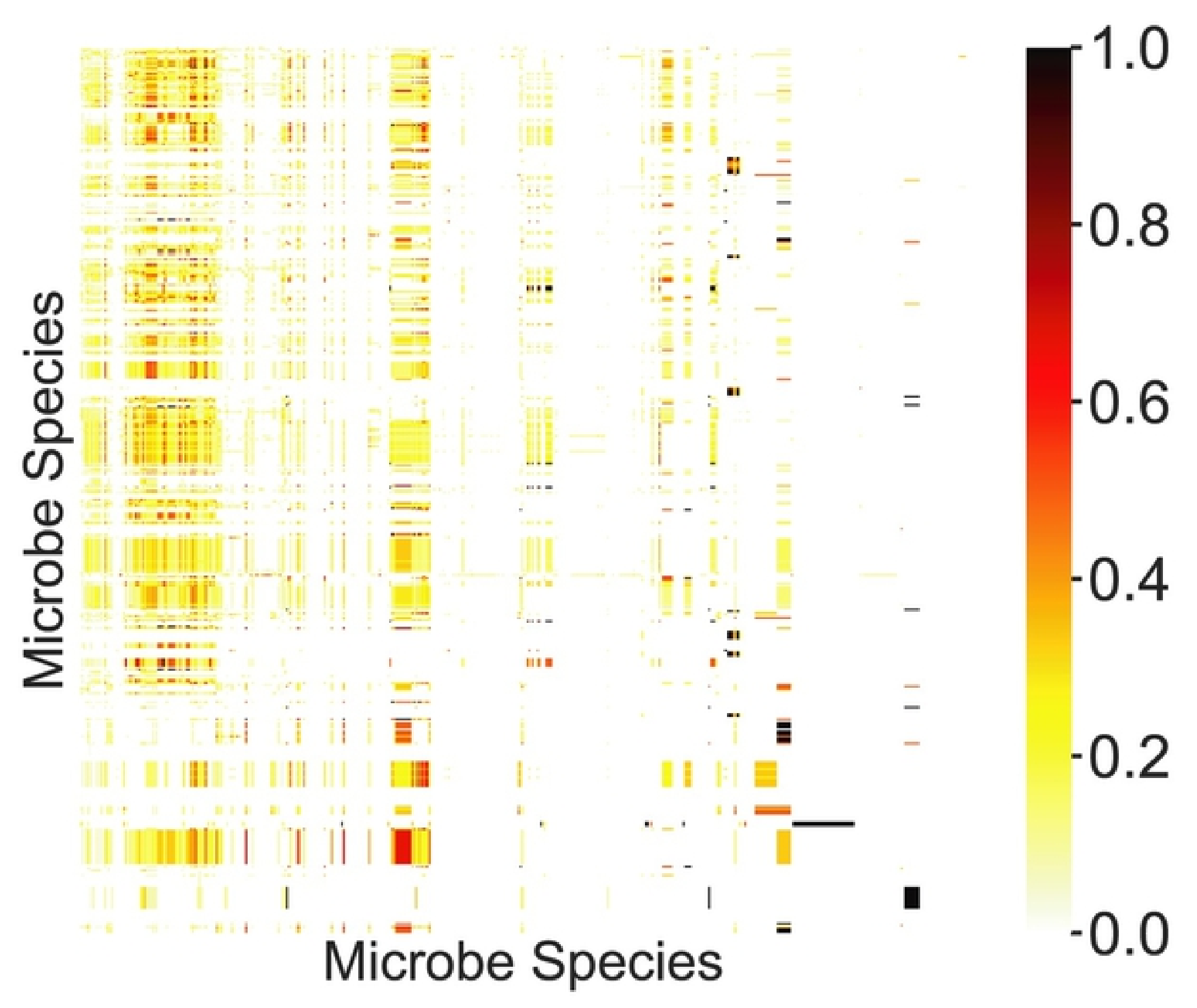

Figure2 
A

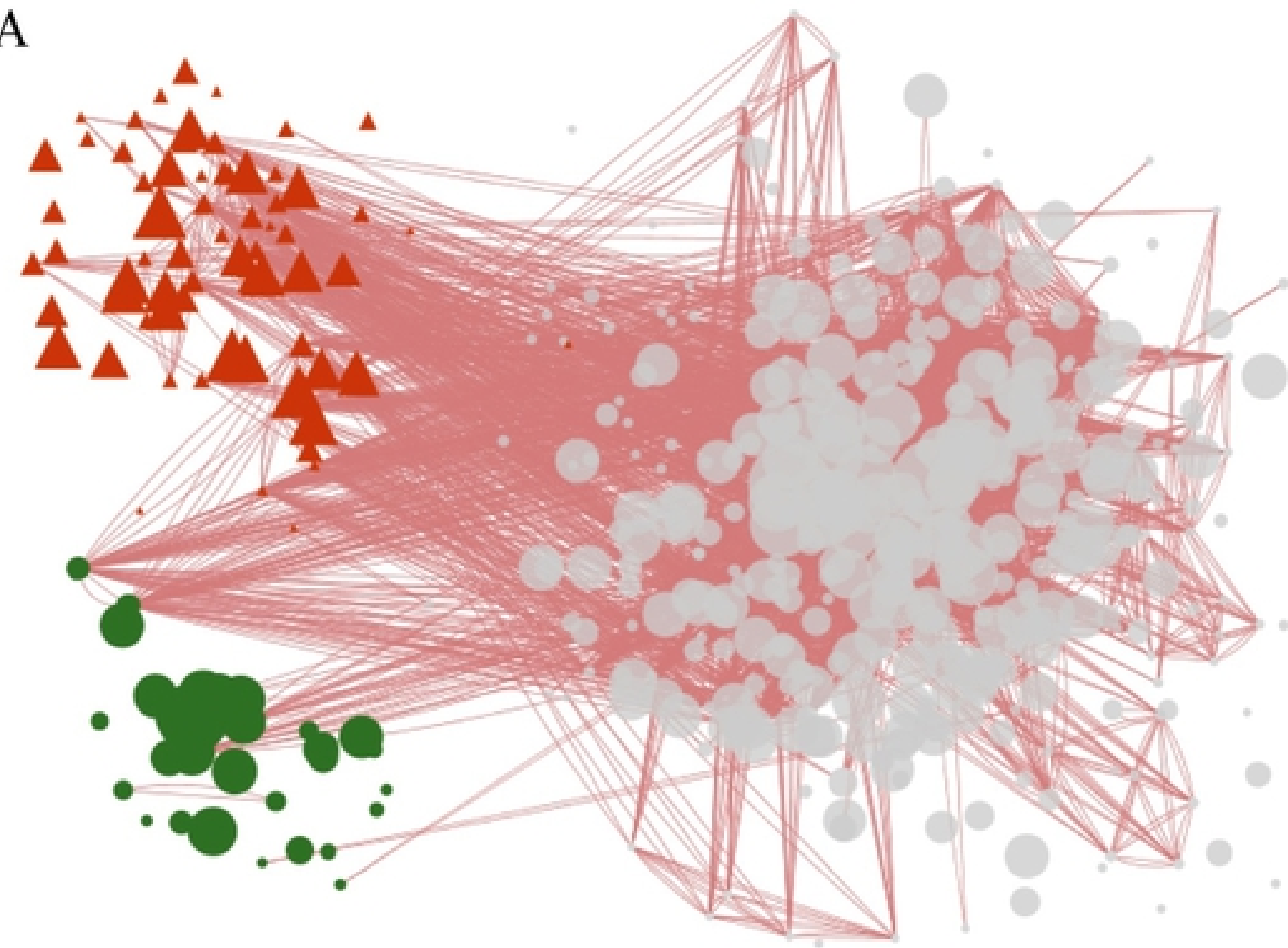

B

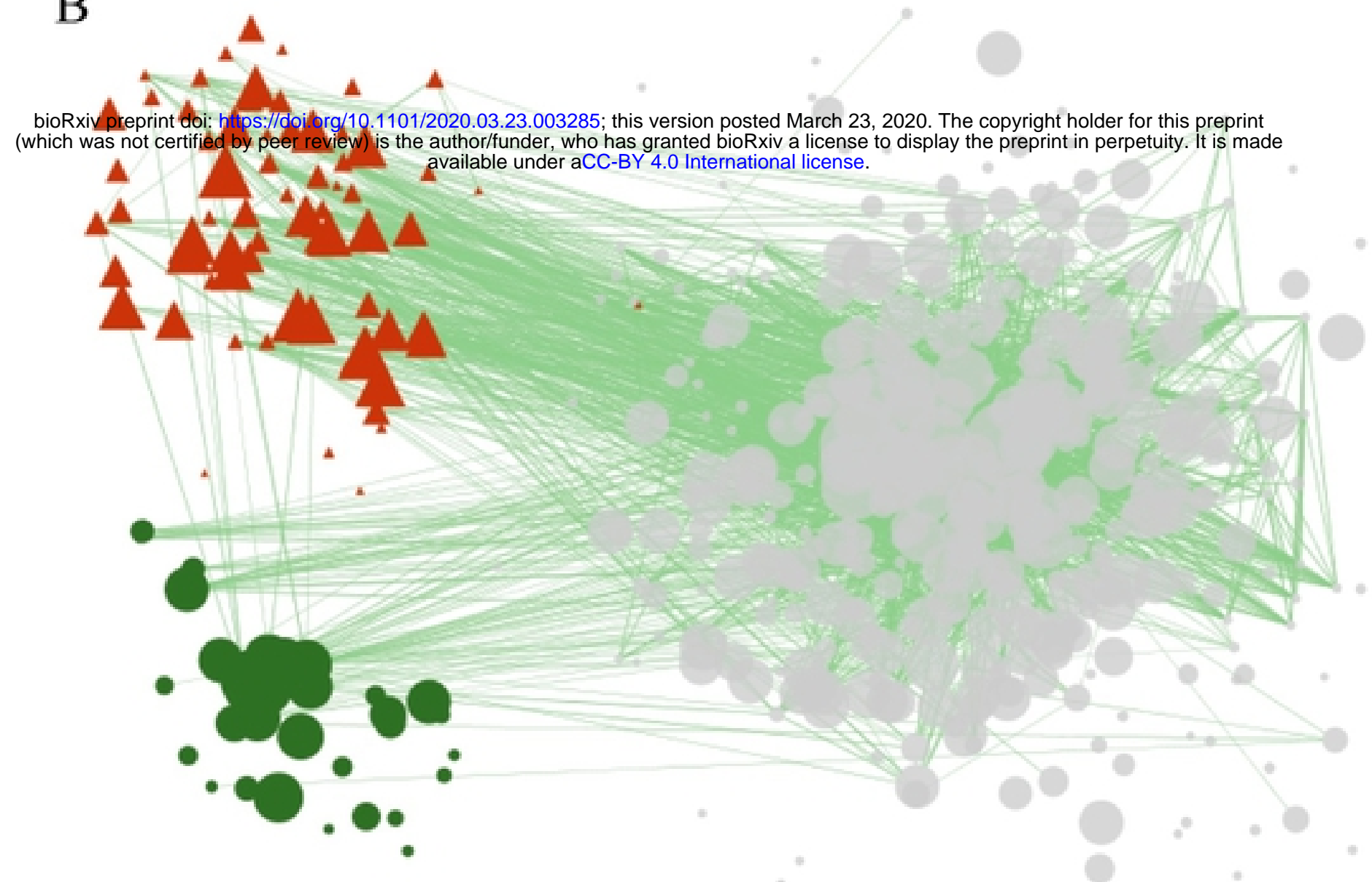

C

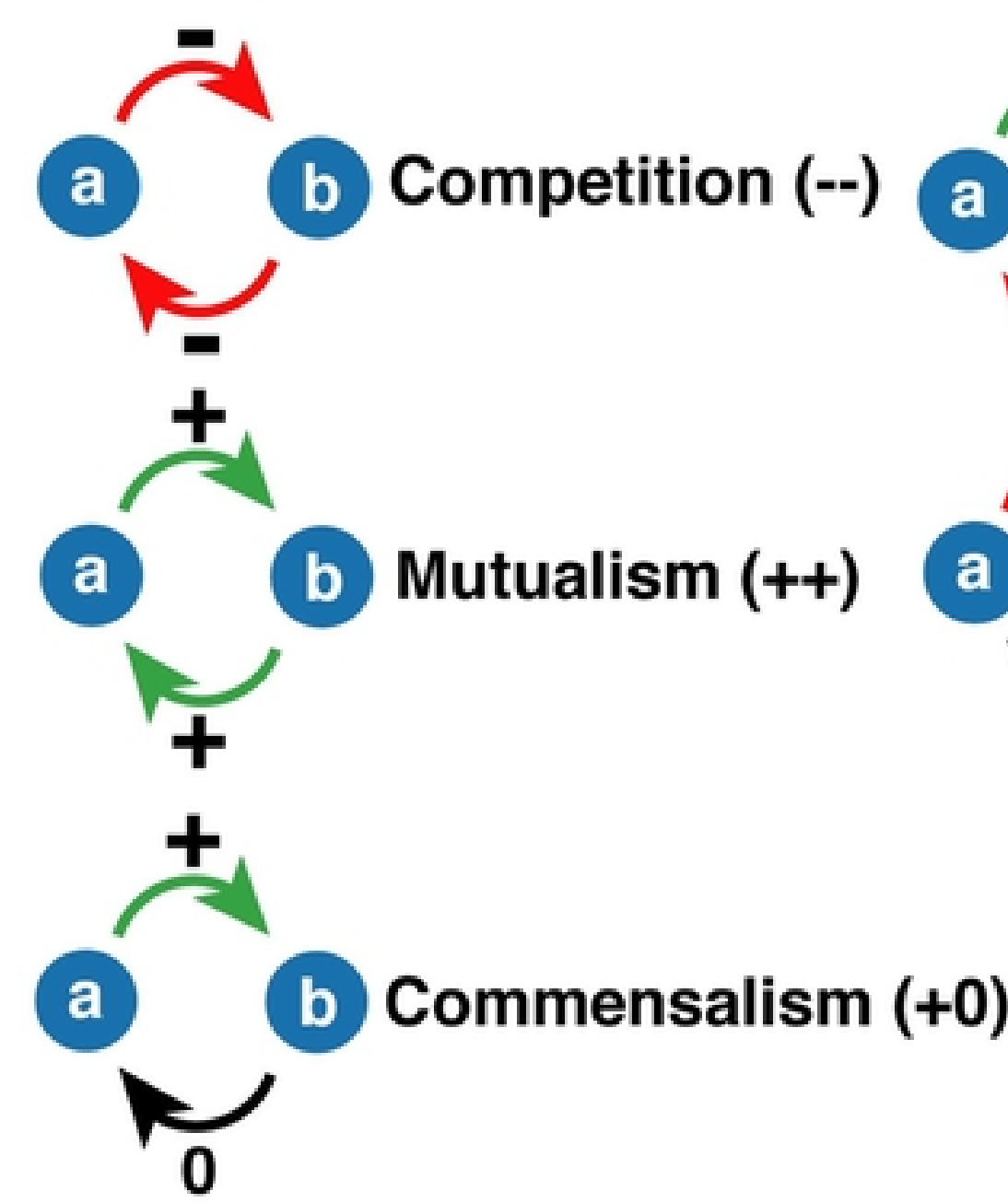

Figure3 
